\title{
CORRESPONDENCE
}

\section{COVID-19 associated pulmonary aspergillosis: regional variation in incidence and diagnostic challenges}

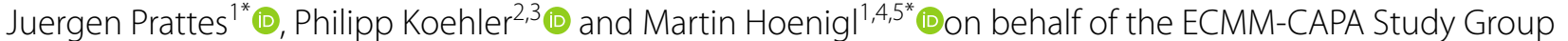

(C) 2021 Springer-Verlag GmbH Germany, part of Springer Nature

We thank Rouze et al. for the thoughtful remarks [1] on our multinational study on pulmonary aspergillosis (CAPA) associated to coronavirus disease 2019 (COVID19) [2].

We agree that diagnosis of CAPA is complex and requires incorporation of clinical presentation, imaging and mycological work-up with the additional challenge of possible diagnostic uncertainty in patients with acute respiratory failure. Further, diagnostic strategies vary widely between centers and systemic CAPA screening is rarely performed, as shown in our study where even serum galactomannan (GM) levels were available in less than half of patients (277/592) [2]. This scarce may be a result of low serum GM sensitivity (19\%) [2] reflecting the primary airway invasive character of the disease with delayed angioinvasion, mirroring pathophysiology of aspergillosis in other non-neutropenic populations. Consequently, mycological evaluation of respiratory specimens is key for early diagnosis and successful management of CAPA. While bronchoalveolar lavage fluid (BALF) has been the benchmark specimen for diagnosing aspergillosis in the non-neutropenic host, BALF samples have not been widely available from COVID-19 patients due to safety concerns in the early phase of the pandemic (BALF was obtained in about half of our cohort [2]). Thus, other respiratory specimens such as non-bronchoscopic

\footnotetext{
*Correspondence: juergen.prattes@medunigraz.at; hoeniglmartin@ gmail.com

1 Division of Infectious Diseases, Department of Internal Medicine, ECMM Excellence Center, Medical University of Graz, Graz, Austria Full author information is available at the end of the article
} \section{Springer \\ 伨}

lavage and tracheal aspiration samples have been widely used for CAPA diagnosis. Although evaluation of clinical specificity was lacking, mycological evidence from these samples - often with GM cutoffs derived from BALF-have also been utilized for classification of CAPA according to modified Blot criteria. Emerging evidence indicates that GM testing from tracheal aspirates may be less discriminative between Aspergillus colonization and CAPA, and specificity may only improve once cutoffs are significantly increased ( $\geq 2.0$ optical density index) [3]. Thereby CAPA classification efforts utilizing Blot criteria may have resulted in an overestimation of the real disease burden. These limitations of variations in early classification and CAPA overestimation where recently overcome by the more conservative 2020 ECMM/ISHAM CAPA consensus definitions. Compared to the modified Blot criteria, application of these new criteria resulted in a reduction of the mean incidence of probable/ proven CAPA cases from $19 \%$ to $11.9 \%$ among evaluable cases[4], bringing the prevalence of CAPA cases closer to that suggested by autopsy studies. As an important strength, our study uniformly applied these more stringent ECMM/ISHAM criteria to all cases, resulting in an overall incidence of probable/proven CAPA of $15.4 \%$ (92/592), although incidence varied widely between participating centers [2]. This wide range may reflect local epidemiological variations that may be COVID-specific (e.g., non-uniform approaches to COVID-19 treatment) but may also be more generalizable, reflecting different burden of Aspergillus exposure, diagnostic accuracy and genetic predisposing risk factors.

Detailed evaluation of our cohort has indicated older age, need for invasive respiratory support and receipt of tocilizumab as independent risk factors associated with 
development of CAPA, and presence of CAPA represented an independent factor for reduced probability of ICU survival, even after controlling for underlying conditions [5].

Importantly our data reflect a real-life scenario with no predefined CAPA screening or fungal diagnostics strategies and despite enrolling prospectively, not all centers had CAPA and non-CAPA patients reported for the entire study period.

\begin{abstract}
Author details
${ }^{1}$ Division of Infectious Diseases, Department of Internal Medicine, ECMM Excellence Center, Medical University of Graz, Graz, Austria. ${ }^{2}$ Department I of Internal Medicine, ECMM Excellence Center, Medical Faculty and University Hospital Cologne, University of Cologne, Cologne, Germany. ${ }^{3}$ Cologne Excellence Cluster on Cellular Stress Responses in Aging-Associated Diseases (CECAD), University of Cologne, 50937 Cologne, Germany. ${ }^{4}$ Division of Infectious Diseases and Global Public Health, University of California San Diego, San Diego, CA, USA. ${ }^{5}$ Clinical and Translational Fungal-Working Group, University of California San Diego, San Diego, CA, USA.
\end{abstract}

\section{Acknowledgements}

ECMM Study Group Authors: Joost Wauters, Daniele Roberto Giacobbe, Katrien Lagrou, Jon Salmanton-García, Riina Rautemaa-Richardson, Stefan Hatzl, Johan Maertens, Yves Debaveye, Marc Bourgeois, Marijke Reynders, Lynn Rutsaert, Niels Van Regenmortel, Piet Lormans, Simon Feys, Alexander Christian Reisinger, Oliver A. Cornely, Tobias Lahmer, Maricela Valerio, Laurence Delhaes, Kauser Jabeen, Joerg Steinmann, Mathilde Chamula, Matteo Bassetti.

\section{Declarations}

\section{Conflicts of interest}

JP has received personal fees from Gilead Sciences and Pfizer, research funding from MSD and is stoke holder of AbbVie Inc and Novo Nordisk. PK is supported by the German Federal Ministry of Research and Education and the State of North Rhine-Westphalia, Germany and has received non-financial scientific grants from Miltenyi Biotec GmbH, Bergisch Gladbach, Germany, and the Cologne Excellence Cluster on Cellular Stress Responses in Aging-Associated Diseases, University of Cologne, Cologne, Germany, and received lecture honoraria from and/or is advisor to Akademie für Infektionsmedizin e.V., Ambu
GmbH, Astellas Pharma, European Confederation of Medical Mycology, Gilead Sciences, GPR Academy Ruesselsheim, MSD Sharp \& Dohme GmbH, Noxxon N.V., and University Hospital, LMU Munich outside the submitted work. MH received research funding from Gilead Sciences, Astellas, Scynexis, F2G and Pfizer.

\section{Publisher's Note}

Springer Nature remains neutral with regard to jurisdictional claims in published maps and institutional affiliations.

Received: 16 August 2021 Accepted: 16 August 2021

Published online: 1 September 2021

\section{References}

1. Rouze A, Lemaitre E, Nseir S (2021) COVID-19-associated pulmonary invasive aspergillosis: high incidence or difficult diagnosis? Intensive Care Med. https://doi.org/10.1007/s00134-021-06499-8

2. Prattes J, Wauters J, Giacobbe DR, Lagrou K, Hoenigl M, Group E-CS (2021) Diagnosis and treatment of COVID-19 associated pulmonary apergillosis in critically ill patients: results from a European confederation of medical mycology registry. Intensive Care Med

3. Roman-Montes CM, Martinez-Gamboa A, Diaz-Lomeli P, CervantesSanchez A, Rangel-Cordero A, Sifuentes-Osornio J, Ponce-de-Leon A, Gonzalez-Lara MF (2021) Accuracy of galactomannan testing on tracheal aspirates in COVID-19-associated pulmonary aspergillosis. Mycoses 64:364-371

4. Fekkar A, Neofytos D, Nguyen MH, Clancy CJ, Kontoyiannis DP, Lamoth F (2021) COVID-19-associated pulmonary aspergillosis (CAPA): how big a problem is it? Clinical microbiology and infection: the official publication of the European Society of Clinical Microbiology and Infectious Diseases

5. Prattes J, Wauters J, Giacobbe DR, Salmanton-Garcia J, Maertens J, Bourgeois M, Reynders M, Rutsaert L, van RegenmortelN, Lormans P, Feys S, Reisinger AC, Cornely OA, Lahmer T, Valerio M, Delhaes L, Jabeen K, Steinmann J, Chamula M,Bassetti M, Hatzl S, Richardson R, Lagrou K, Hoenigl M (2021) Risk factors and outcome of pulmonary aspergillosis in critically ill coronavirus disease 2019 patients - a multinational observational study by the European Confederation of Medical Mycology.Clinical microbiology and infection : the official publication of the European Society of Clinical Microbiology and InfectiousDiseases. https://doi.org/10.1016/j. cmi.2021.08.014 